\title{
Self-reported physical functional health predicts future bone mineral density in EPIC-Norfolk cohort
}

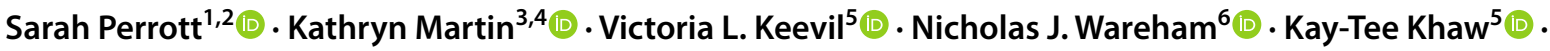 \\ Phyo Kyaw Myint ${ }^{1,2}$ (])
}

Received: 22 July 2021 / Accepted: 17 November 2021 / Published online: 28 January 2022

(C) International Osteoporosis Foundation and National Osteoporosis Foundation 2021

\begin{abstract}
Summary Using a large population sample from the UK, we found that self-reported physical functional health may be used to predict future bone mineral density especially in women. It may be a useful and inexpensive way to identify individuals before further decline in bone mineral density and the risk of fracture.

Purpose Self-reported physical functional health may predict bone mineral density (BMD) and thus provide a method to identify people at risk of low BMD. In this study, the association between the 36-item short-form questionnaire (SF-36) physical component summary (PCS) score and future BMD in participants aged 40-79 years enrolled in the European Prospective Investigation of Cancer-Norfolk study was investigated.

Methods Associations between a participant's SF-36 PCS score, measured 18 months after baseline health check, and broadband ultrasound attenuation (BUA-a measure of BMD), measured 2-5 years after baseline, were examined using sex-specific linear and logistic regression analyses adjusting for age, BMI, medical co-morbidities, lifestyle and socioeconomic factors.

Results Data from 10,203 participants, mean (standard deviation (SD)) age 61.5 (8.9) years (57.4\% women), were analysed from 1993 to 2000. For every five points lower PCS score in men and women, there was approximately a $0.5 \mathrm{~dB} / \mathrm{MHz}$ lower mean BUA. In women, a PCS score of less than one standard deviation (1SD) below the sex-specific mean was associated with having a low BUA $(<1 \mathrm{SD}$ below sex-specific mean) and very low BUA $(<2.5 \mathrm{SD}$ below the sex specific mean); odds ratio (OR) (95\% confidence interval) $1.53(1.24,1.88)$ and $8.28(2.67,25.69)$, respectively. The relationship was lesser so in men; corresponding OR $(95 \% \mathrm{CI})$ were $1.34(0.91,1.98)$ and $2.57(0.72,9.20)$, respectively.
\end{abstract}

Conclusions Self-reported physical functioning predicts BMD in an apparently healthy population, particularly in women. This could potentially provide an inexpensive, simple screening tool to identify individuals at risk of osteoporosis.

Keywords Physical functioning $\cdot$ Bone mineral density $\cdot$ Older adults $\cdot$ SF-36

Phyo Kyaw Myint

phyo.myint@abdn.ac.uk

1 Ageing Clinical \& Experimental Research (ACER)

Team, Institute of Applied Health Sciences, University of Aberdeen, Aberdeen, UK

2 School of Medicine, Medical Sciences and Nutrition, University of Aberdeen, Polwarth Building, Room 4:013, Foresterhill, Aberdeen AB25 2ZD, UK

3 Institute of Applied Health Sciences, University of Aberdeen, Aberdeen, UK

4 The Aberdeen Centre for Arthritis and Musculoskeletal Health, Aberdeen, UK

5 Department of Medicine, University of Cambridge, Cambridge, UK

6 MRC Epidemiology Unit, Cambridge, UK

$\begin{array}{ll}\text { Abbreviations } \\ \text { BMD } & \text { Bone mineral density } \\ \text { BMI } & \text { Body mass index } \\ \text { BUA } & \text { Broadband ultrasound attenuation } \\ \text { CI } & \text { Confidence interval } \\ \text { COAD } & \text { Chronic obstructive airways disease } \\ \text { CUBA } & \text { Calcaneal ultrasound broadband attenuation } \\ \text { DEXA } & \text { Dual-energy X-ray absorptiometry } \\ \text { EPIC } & \text { European Prospective Investigation into Cancer } \\ \text { FRAX } & \text { Fracture risk assessment tool } \\ \text { HLEQ } & \text { Health and life experiences questionnaire } \\ \text { HRQL } & \text { Health-related quality of life } \\ \text { HRT } & \text { Hormone replacement therapy } \\ \text { OR } & \text { Odds ratio } \\ \text { PCS } & \text { Physical component summary }\end{array}$


SD Standard deviation

SE Standard error

SF-36 Short-form 36

TDI Townsend deprivation index

WHO World Health Organisation

2HC Second health check

\section{Introduction}

Osteoporosis is a major public health issue [1], with one in three women experiencing an osteoporotic fracture in their lifetime [2]. Osteoporosis is a silent disease until fracture occurrence, the latter associated with poor outcomes in the ageing population $[1,3]$. Besides limiting mobility and independence, the disability caused by osteoporosis is a substantial economic burden and contributor to healthcare system pressures, estimated to annually cost up to $\$ 25.3$ billion in the USA [4-6]. Early detection of poor bone health and prevention of osteoporosis are more effective than relying on pharmaceutical intervention in later life [3].

Various health-related quality of life (HRQL) measures have been used to predict future health outcomes. Selfreported functional health measured by the short-form 36-item questionnaire (SF-36) is used to assess HRQL, and previous studies have shown scores to be predictive of many outcomes-including mortality and stroke risk [7-10]. Weight-bearing physical activity is a sustainable method to enhance bone health, preserve bone mineral density (BMD) and enable bone regeneration $[1,3,6]$. Since lifestyle is a determining factor of SF-36, self-reported physical functioning measures could prove a simple and inexpensive method to identify people at risk of low BMD.

Bone mass is generated in adolescence; failure to reach and maintain peak BMD during adulthood is a crucial factor in determining future osteoporosis risk $[1,3,6]$. Women are disproportionately impacted by osteoporosis due to hormonal changes at menopause, with osteoporotic fractures being more common than stroke, myocardial infarction and breast cancer combined $[11,12]$. Exercise can maintain BMD in those aged over 60 years, although beyond this age BMD is harder to regain $[3,13]$. One in eight men will have an osteoporotic fracture in their lifetime; despite less rapid bone deterioration compared to women, fracture-related mortality can be twice as high $[14,15]$.

The relationship between physical function and BMD is well-established [16]; multiple physical performance measures (e.g. walking speed, grip strength) have proven to be of high predictive value regarding future fracture risk [17]. Identifying poor BMD before further decline and fracture occurrence is imperative; therefore, indicators of poorer BMD provided by self-reported physical functional health could help counter this public health issue.
Low self-reported health-related quality of life measures have previously been associated with osteoporosis [18, 19]. In this study, physical functional health assessed using SF-36 was used to predict future BMD in men and women aged $40-79$ years.

\section{Material and methods}

\section{Population}

This study population consists of 12,071 participants who took part in the European Prospective Investigation of Cancer-Norfolk (EPIC-Norfolk) cohort study who had their BMD evaluated. EPIC-Norfolk participant recruitment and methodology have previously been described [20]. In brief, participants were identified from general practice age-sex registers and invited to participate in a baseline questionnaire, health check, nutrition evaluation and biospecimen collection from 1993 to 1997 . The cohort was followed up over 25 years with health checks at regular intervals. The EPIC-Norfolk cohort are representative of the UK population, albeit with lower proportion of smokers [20].

\section{Predictor variables}

Participants were asked to complete a health and life experiences questionnaire (HLEQ) 18 months after baseline. This questionnaire included the anglicised short-form 36 (UK SF-36), which measures self-reported health across eight domains: perceptions of general health; social functioning; physical functioning; role limitation due to physical issues; role limitation due to emotional issues; mental health; pain; energy and vitality [21]. Overall scores were calculated for each domain and then converted to a 0-100 scale, where 0 represents poor and 100 represents best possible health $[21,22]$. The physical component summary (PCS) score is derived using all eight SF-36 domains [21]. In this study, PCS was used as a continuous predictor variable (every five-point decrease in PCS) and as a categorical variable. Participants were categorised into those with poor physical functioning, a PCS score of less than one standard deviation (SD) below the sex-specific mean, and those with good physical functioning, a PCS score greater than this.

\section{Outcome variables}

BMD was assessed using broadband (i.e. calcaneal) ultrasound attenuation (BUA) measured at the second health check (2HC) from 1998 to 2000, at least 3 years after baseline, using a contact ultrasound bone analyser (CUBA) device [23]. Cross-sectional analysis approach was employed since the outcome is a measurement as opposed to an 
incident event such as heart attack and thus not appropriate for Cox-regression models. BUA can discriminate patients with low BMD equally as well as dual-energy X-ray absorptiometry (DEXA) [24]. BUA measures were taken at least twice in each foot and the mean BUA from left and right were used for analysis. A greater BUA reading is indicative of higher BMD. Each CUBA device was calibrated daily and calibration between all devices used for this study was checked monthly [25].

Participants with a mean BUA less than 1SD below the sex-specific mean were considered to have low BMD. Participants with a mean BUA less than 2.5SD below the sex-specific mean were considered to have very low BMD. These cut-offs were based on evidence which suggests for each SD decrease below the young-adult mean, fracture risk doubles [12, 26, 27]. Although our study uses the means of older rather than young adults, this measure should identify individuals with at least osteopenia $(\mathrm{BMD}<1 \mathrm{SD}$ below young-adult mean), as per the World Health Organisation (WHO) definition [27]. Participants with a BUA less than 2.5SD below the sex-specific mean, classified as having very low BMD, were likely to have osteoporosis based on the WHO definition of osteoporosis (BMD $<2.5 \mathrm{SD}$ below young-adult mean) [27].

\section{Covariates}

Age, sex, weight and height were recorded and body mass index (BMI) was calculated at $2 \mathrm{HC}$ using a standardised protocol [28]. Cigarette smoking status was obtained and recorded as 'current', 'former' or 'never' smoker, which was reclassified into 'former or current smoker' and 'never smoker' for the purpose of this study. Similarly, average weekly alcohol consumption was recorded and re-classified into 'consumes alcohol regularly' (at least one drink a week) or 'minimal or non-drinker'.

Participant socioeconomic status was evaluated by three measures: social class; education status and Townsend deprivation index (TDI). Social class was categorised into five groups according to participant's own or partner's occupation, where social class I consisted of professionals, and social class V included unskilled manual workers [20]. Educational status was split into four groups based on highest qualification achieved (i.e. degree or equivalent; A-level or equivalent; O-level or equivalent and less than O-level). O-level usually indicates academic achievement to the minimum UK school leaving age. TDI is a multi-factor scoring system used to determine deprivation, where 0 is the national mean, with positive scores representing greater deprivation and negative scores representing lower deprivation [29]. Social class was reclassified into 'semi/unskilled workers' (social classes IV and V) and 'skilled workers' (social classes I, II and III). Highest education level achieved was reclassified into 'none or O-level' and 'A-level or degree', and TDI was classified into ' 0 or less' and 'above 0 '. These reclassifications were made as deprivation, low social class and education status tend to be associated with suboptimal BMD [30, 31].

Participants were asked in the baseline health questionnaire to self-report any medically diagnosed illnesses (e.g. cancer, diabetes mellitus, depression requiring treatment, stroke and myocardial infarction). Use of hormone replacement therapy (HRT) at any time and steroid use at $2 \mathrm{HC}$ were also recorded.

\section{Statistical analyses}

Analyses were performed using SPSS version 26.0 (IBM, New York, USA). Participants with prevalent cancer and who did not have either a BUA reading or PCS score were excluded. Only participants with data for all the covariates required for the models were included. All analyses were stratified by sex as previous studies show sex-specific differences in BMD [26].

Participant characteristics were described using proportions, means and medians. Differences between men and women were compared using the $t$-test or Mann-Whitney test for continuous variables, or the Pearson chi-squared test for categorical variables.

Sex-specific linear regression models were used to explore associations between PCS score (every 5 point decrease) and BUA as a continuous outcome measure. Multivariate models were used to examine whether potential confounders may attenuate any relationships observed. In men, we first adjusted for the following: ever smoked; steroid use at 2HC; education level below A-levels; social class; alcohol consumption; depression requiring treatment; BMI and age. In women, we adjusted for the same confounders as men, but also HRT use. Confounders were identified using the Pearson chi-squared test and selected if they showed association with BUA and PCS score. The unstandardised $\beta$-coefficients were calculated with the standard of error (SE) for each 5 points decrease in PCS score and presented with $p$ values.

Relationships between poor physical functioning, defined as participants with a PCS score $<1$ SD below the sex-specific mean, and low BUA $(<1$ SD below the sex-specific mean) and very low BUA $(<2.5 \mathrm{SD}$ below the sex specific mean) were also explored using logistic regression, adjusting for the same confounders as in the linear regression models. Odds ratios (OR) were presented with $95 \%$ confidence intervals $(95 \% \mathrm{CI})$ and associated $p$ values.

Although the relationship between PCS score and BUA is longitudinal, we used cross-sectional analysis (OR) as both predictor and outcome were not discrete episodes, but 
measurements which could remain stable and more likely to deteriorate subtly over time.

\section{Results}

\section{Main findings}

Of the 15,786 participants who attended $2 \mathrm{HC}, 11,995 \mathrm{had}$ BUA measured. Of these, 536 were missing SF-36 PCS scores and remaining participants with a cancer diagnosis $(n=747)$ were excluded. Missing data were more likely in women and in those aged over 65 years; $5.1 \%$ of participants had missing data in at least one covariable: smoking status $(n=83)$, highest education level attained $(n=5)$, BMI $(n=20)$, social class $(n=5)$, alcohol consumption status $(n=209)$, ever taken HRT $(n=132)$ and self-reported depression $(n=896)$. The final sample included 10,203 participants (57.1\% women), mean (SD) age 61.5 (8.9) years, who had a complete dataset, detailed in Table 1.

Table 1 shows the distribution of sample characteristics by sex. The population is predominantly non-deprived, of
Table 1 Characteristics of 4378 men and 5825 women EPICNorfolk participants at $2 \mathrm{HC}$, with exception of SF-36 which was measured at HLEQ1

\begin{tabular}{|c|c|c|c|}
\hline Characteristics & Men & Women & $p$ value \\
\hline Number (\%) & 4378 & 5825 & \\
\hline \multicolumn{4}{|l|}{ Smoking status } \\
\hline Current smoker & $307(7.0)$ & $435(7.5)$ & \multirow[t]{3}{*}{$<0.001^{\mathrm{a}}$} \\
\hline Former smoker & $2384(54.5)$ & $1849(31.7)$ & \\
\hline Never smoker & $1687(38.5)$ & $3541(60.8)$ & \\
\hline \multicolumn{4}{|l|}{ Occupation } \\
\hline Unskilled/semi-skilled & $1589(36.3)$ & $1994(34.2)$ & \multirow[t]{2}{*}{$0.026^{\mathrm{a}}$} \\
\hline Skilled & $2789(63.7)$ & $3831(65.8)$ & \\
\hline \multicolumn{4}{|l|}{ Highest education status } \\
\hline Degree & 785 (17.9) & $792(13.6)$ & \multirow[t]{3}{*}{$<0.001^{\mathrm{a}}$} \\
\hline A-levels & $2082(47.6)$ & $2226(38.2)$ & \\
\hline O-levels or lower & $1502(34.3)$ & $2800(48.1)$ & \\
\hline Deprivation (Townsend index $>0$ ) & $655(15.0)$ & $874(15.0)$ & $0.065^{\mathrm{a}}$ \\
\hline \multicolumn{4}{|l|}{ Self-reported prevalent illness } \\
\hline Any & $1769(40.4)$ & $2554(43.8)$ & \multirow[t]{6}{*}{$<0.001^{\mathrm{a}}$} \\
\hline Cardiovascular disease & $328(7.5)$ & $177(3.0)$ & \\
\hline Diabetes & $174(4.0)$ & $129(2.2)$ & \\
\hline COAD & $771(17.6)$ & $1256(21.6)$ & \\
\hline Arthritis & $1034(23.6)$ & $1920(33.0)$ & \\
\hline Depression requiring treatment & $416(9.5)$ & $1078(18.5)$ & \\
\hline \multicolumn{3}{|l|}{ Consumes alcohol regularly } & \multirow[t]{3}{*}{$<0.001^{\mathrm{a}}$} \\
\hline Yes & $3872(88.4)$ & $4674(80.2)$ & \\
\hline No & $434(9.9)$ & $1014(17.4)$ & \\
\hline Steroid use at $2 \mathrm{HC}$ & $168(3.8)$ & $297(5.1)$ & $0.001^{\mathrm{a}}$ \\
\hline Ever used HRT & - & $2276(39.1)$ & \\
\hline \multicolumn{4}{|l|}{ Mean (SD) } \\
\hline Age in years & $62.3(8.9)$ & $61.0(8.9)$ & $0.867^{\mathrm{b}}$ \\
\hline $\mathrm{BMI}$ in $\mathrm{kg} / \mathrm{m}^{2}$ & $26.8(3.3)$ & $26.5(4.4)$ & $<0.001^{\mathrm{b}}$ \\
\hline BUA averaged over both heel bones in $\mathrm{dB} / \mathrm{MHz}$ & $90.2(17.3)$ & $72.5(16.4)$ & $<0.001^{\mathrm{b}}$ \\
\hline \multicolumn{4}{|l|}{ Median (IQR) } \\
\hline Alcohol consumption in units/week & $6.5(2.0,14.0)$ & $2.0(1.0,7.0)$ & $<0.001^{\mathrm{c}}$ \\
\hline SF-36 PCS score & $51.5(44.8,55.2)$ & $51.1(43.6,55.2)$ & $0.015^{\mathrm{c}}$ \\
\hline SF-36 MCS score & $55.8(50.3,58.9)$ & $54.7(48.1,58.4)$ & $<0.001^{\mathrm{c}}$ \\
\hline
\end{tabular}

${ }^{\mathrm{a}} p$ value as calculated by chi-squared test, ${ }^{\mathrm{b}}$ independent samples $t$-test and ${ }^{\mathrm{c}}$ Mann-Whitney test

Abbreviations: 2HC, second health check, between 1997 and 2000; SF36, 36-item short-form questionnaire; HLEQ1, health and lifestyle questionnaire, taken 18 months after first health check between 1994 and 1998; $C O A D$, chronic obstructive airway disease; $H R T$, hormone replacement therapy; $S D$, standard deviation; $B M I$, body mass index; $B U A$, broadband ultrasound attenuation, averaged over both heel bones at $2 \mathrm{HC}$ measured in $\mathrm{dB} / \mathrm{MHz} ; P C S$, physical component summary; $M C S$, mental component summary 
high educational and occupational status, and non-obese. PCS score was on average 0.4 points lower in women compared to men $(p=0.015)$, with BUA greater in men $(p<0.001)$, with mean (SD) BUA 90.2 (17.3) dB/MHz and 72.5 (16.4) dB/MHz, respectively.

\section{PCS score as a continuous predictor of BUA}

Table 2 shows the sex-specific linear regression models for PCS score as a predictor of BUA. In the unadjusted analysis in men, a 5-point lower PCS score corresponds with $0.52 \mathrm{~dB}$ / $\mathrm{MHz}$ lower mean BUA. The adjusted model for confounders had $\beta$-coefficient (SE) 0.47 (0.03) $(p=0.001)$. In women, similar changes in BUA for every 5-point lower PCS score were observed in the unadjusted analyses, but associations weakened in the multivariate model ( $\beta$-coefficients (SE) 0.59 (0.02) and $0.19(0.02)$, respectively).

\section{PCS score as a categorical predictor of low and very Iow BUA}

Table 3 shows the OR and 95\% CI representing associations between poor physical functioning (PCS score $<1 \mathrm{SD}$ below sex-specific mean) and low BUA, indicative of at least osteopenia [27]. The same variables were controlled for as in the linear regression model. There were 425 women and 235 men with low BUA, compared to 5400 women and 4143 men with normal BUA. In women, consistent associations between poor physical functioning and higher odds of low BUA were found across unadjusted and adjusted models, with an OR $(95 \% \mathrm{CI})$ of $1.53(1.24,1.88)$ in the multivariable model. In men, the same direction of association was observed between poor physical functioning and low BUA,

Table 2 The $\beta$-coefficient (SE) of linear regression model for change in BMD measure by every 5-point decrease in SF-36 PCS score in 4378 men and 5825 women, with and without adjustment

\begin{tabular}{llr}
\hline & $\beta$-coefficient (SE) & $p$ value \\
\hline Men & & \\
$\quad$ Unadjusted & $-0.52(0.03)$ & $<0.001$ \\
$\quad$ Adjusted for confounders & $-0.47(0.03)$ & 0.001 \\
Women & & \\
$\quad$ Unadjusted & $-0.59(0.02)$ & $<0.001$ \\
$\quad$ Adjusted for confounders & $-0.19(0.02)$ & 0.056 \\
\hline
\end{tabular}

Confounders: ever smoked; steroid use at $2 \mathrm{HC}$; education level below A-levels; social class based on skilled occupation; alcohol consumption; depression requiring treatment; ever used HRT (women only); BMI; age

Abbreviations: PCS, physical capability score, part of SF-36 (36-item short-form questionnaire), score $0-100$-where 100 is peak physical health; $S E$, standard error; $n$, number included in analysis; $B U A$, broadband ultrasound attenuation, measured in $\mathrm{dB} / \mathrm{MHz}$, measure of bone mineral density
Table 3 The odds ratio (95\% CI) of low BMD defined as BMD measure less than-1SD value below sex-specific mean for those with SF-36 PCS values less than-1SD, compared to their counterparts with higher SF-36 PCS score, in 4378 men and 5825 women, with and without adjustment

Odds ratio $(95 \% \mathrm{CI}) \quad p$ value

Men

Mean (SD) BUA $=90.22(17.29) \mathrm{dB} / \mathrm{MHz}$

$\begin{array}{lll}\text { Unadjusted } & 1.39(0.96,2.02) & 0.083 \\ \text { Adjusted for confounders } & 1.34(0.91,1.98) & 0.140\end{array}$

Women

Mean (SD) BUA = $72.52(16.42) \mathrm{dB} / \mathrm{MHz}$

$\begin{array}{lll}\text { Unadjusted } & 1.72(1.42,2.07) & <0.001 \\ \text { Adjusted for confounders } & 1.53(1.24,1.88) & <0.001\end{array}$

Confounders in men: ever smoked $(p=0.283)$; steroid use at $2 \mathrm{HC}$ $(p=0.496)$; education level below A-levels $(p=0.532)$; social class based on skilled occupation $(p=611)$; alcohol consumption $(p=0.207)$; depression requiring treatment $(p=0.855)$; BMI $(p=0.036)$; age $(p=0.052)$

Confounders in women: ever smoked $(p=0.385)$; steroid use at $2 \mathrm{HC}(p=0.125)$; education level below A-levels $(p=0.011)$; social class based on skilled occupation $(p=0.299)$; alcohol consumption $(p=0.029)$; ever used HRT $(p<0.001)$; depression requiring treatment $(p=0.260)$; BMI $(p<0.001)$; age $(p<0.001)$

Abbreviations: $S F-36,36$-item short-form questionnaire; $P C S$, physical component summary; $S D$, standard deviation; $C I$, confidence interval; $B U A$, broadband ultrasound attenuation, measured in $\mathrm{dB} /$ $\mathrm{MHz}$, measure of bone mineral density

but associations were not significant (multivariable model: OR $1.34,95 \%$ CI $0.91,1.98$ ).

Similarly, Table 4 shows the OR and 95\% CI representing relationships between poor PCS and very low BUA, indicative of osteoporosis [27]. In this analysis, there were 11 women and 5 men classified as having very low BUA. Strong associations between poor physical functioning and higher odds of very low BUA were observed in women, although CIs were wide, with an OR (95\% CI) of 8.28 $(2.67,25.69)$ in the adjusted model. The association was less pronounced in men and, although the same trends were observed, results did not reach statistical significance (fully adjusted model OR: $2.57,95 \%$ CI $0.72,9.20$ ).

\section{Discussion}

\section{Main findings}

We found that among EPIC-Norfolk cohort participants, lower PCS scores were associated with poorer future BUA, an indicator of BMD, independent of age, BMI and other confounding factors (i.e. cigarette smoking, steroid use, lower education level, higher alcohol consumption, depression requiring treatment and HRT usage among women). 
Table 4 The odds ratio $(95 \% \mathrm{CI})$ of very low BMD defined as BMD measure less than-2.5SD value below sex-specific mean for those with SF-36 PCS values less than-1SD, compared to their counterparts with higher SF-36 PCS score, in 4378 men and 5825 women, with and without adjustment

Odds ratio $(95 \% \mathrm{CI}) \quad p$ value

\begin{tabular}{lcr}
\hline & Odds ratio (95\% CI) & $p$ value \\
\hline Men & & \\
Mean (SD) BUA = 90.22 (17.29) $\mathrm{dB} / \mathrm{MHz}$ & \\
$\quad$ Unadjusted & $3.82(1.32,11.06)$ & 0.013 \\
$\quad$ Adjusted for confounders & $2.57(0.72,9.20)$ & 0.148 \\
Women & & \\
Mean (SD) BUA =72.52 (16.42) dB/MHz & \\
$\quad$ Unadjusted & $11.49(3.92,33.70)$ & $<0.001$ \\
$\quad$ Adjusted for confounders & $8.28(2.67,25.69)$ & $<0.001$ \\
\hline
\end{tabular}

Confounders in men: ever smoked $(p=0.807)$; steroid use at $2 \mathrm{HC}$ $(p=0.092)$; education level below A-levels $(p=0.187)$; social class based on skilled occupation ( $p=0.552)$; alcohol consumption $(p=0.314)$; depression requiring treatment $(p=0.994) ; \mathrm{BMI}$ $(p=0.478)$; age $(p=0.035)$

Confounders in women: ever smoked $(p=0.962)$; steroid use at $2 \mathrm{HC}(p=0.025)$; education level below A-levels $(p=0.711)$; social class based on skilled occupation $(p=0.811)$; alcohol consumption $(p=0.054)$; ever used HRT $(p=0.745)$; depression requiring treatment $(p=0.299)$; BMI $(p=0.129)$; age $(p=0.041)$

Abbreviations: $S F-36,36$-item short-form questionnaire; $P C S$, physical component summary; $S D$, standard deviation; $C I$, confidence interval; $B U A$, broadband ultrasound attenuation, measured in $\mathrm{dB} /$ $\mathrm{MHz}$, measure of bone mineral density

However, even after adjustment for these, clear differences in BUA between those with higher and lower PCS scores remained. These findings coincide with evidence that higher physical activity in middle-late adulthood is protective against rapid deterioration in bone health $[3,6]$. Another study of EPIC-Norfolk participants found that more time spent doing high-impact physical activity was strongly and positively associated with superior BUA-independent of confounding variables [25]. Several other cohort studies have found physical capability to be a strong indicator of BMD [1, 6, 32-34].

Our results suggested differences in the relationship between low PCS and BUA in men compared to women. When both PCS and BUA were considered as continuous predictor and outcome variables, a strong association between PCS and BUA was evident in men but less so in women. Conversely, when both were considered as categorical variables, and relationships between self-reported physical functioning and low or very low BUA were considered, relationships were stronger in women. Changes in physical functioning in men may be a stronger marker of osteoporosis than in women; however, in this cohort few men had low or very low BUA and results were thus underpowered. As a group, women are more likely to have low BMD; hence, categorical outcomes showed a stronger relationship for women. Other studies investigating physical function measures and BUA have noticed a stark difference between sex $[25,35]$. Jakes et al. found high physical activity in men conferred a BUA of around $9.5 \%$ higher than that of those who reported low physical activity. However, in women this was only $3.4 \%$ higher and similar in size to that of a 4-year age-difference [25]. This is likely to be attributed to higher BMI in women with a sedentary lifestyle, which is protective against rapid BMD deterioration after menopause due to increased oestrogens production by adipose tissue and greater mechanical loading $[25,36]$. A Brazilian study found the impact of prolonged sedentary time and lower physical activity on BMD varied greatly between men and women, with benefits of shorter bouts of sedentary behaviour only observed in women [35].

Our findings suggest that a PCS score of less than 1SD below the sex-specific mean was a weak to moderate predictor of low BUA, indicative of at least osteopenia. Osteopenia is BMD of 1 to $2.5 \mathrm{SD}$ below mean peak levels (i.e. young-adult BMD) and describes low, but not yet critical, bone mass [27, 37]. Early detection and management of osteopenia, including increased weight-bearing exercise and increasing calcium and vitamin D intake, has been demonstrated to reduce fracture risk and improve quality of life [27, 37]. When the outcome was low or very low BUA, indicative of osteopenia or osteoporosis, low PCS was demonstrated to be a strong predictor, although more so in women. This is likely to be due to increased chance of low BMD among women compared to men in the cohort. The number of individuals with very low BUA, particularly in men, was small which resulted in underpowered results.

\section{Clinical relevance}

Older adults constitute the world's fastest growing population, making osteoporosis a significant social and economic burden [12]. Therefore, risk assessing this population is crucial, and although fracture risk assessment tool (FRAX) is an effective tool for older white women, it is less effective among men and non-Caucasians [38]. Furthermore, FRAX assesses fracture risk, not overall bone health status [38]. Indicators of poorer BMD provided by self-reported physical functional health could make a significant contribution to tackle this public health issue. Furthermore, the SF-36 can potentially predict numerous measures besides BMD status, from surgical outcomes $[7,8]$ to risk of death from heart disease and stroke $[9,10]$, emphasising the versatility of the tool as a measure of health status and risk.

\section{Strengths}

To our knowledge, this study is the first to investigate selfreported physical functional health as a predictor of future 
BMD in older adults, allowing for early identification of people at risk of suboptimal BMD potentially earlier than FRAX can [38]. We used a large population-based cohort with validated follow-up methods [20], with the ability to control for a range of confounders including sociodemographic and lifestyle factors. Data were collected prospectively, minimising potential for recall bias, and follow-up was over a critical period of time in the participants' lives when BMD typically deteriorates. This highlighted the effectiveness of PCS score as an early, inexpensive and non-invasive indicator of BMD and therefore risk of future osteopenia and osteoporosis.

\section{Limitations}

The existence of healthy volunteer bias is possible given that EPIC-Norfolk is a volunteer study consisting of predominantly white, middle-class and health-conscious individuals. However, previous literature suggest that EPIC-Norfolk sample characteristics are representative of the UK population [20] and mean SF-36 scores are comparable to mean scores in other population-based studies [39]. As a secondary data analysis of an observational study, there may be unknown confounders that were not adjusted for. Potential confounders measured at baseline or $2 \mathrm{HC}$ may vary during follow-up, e.g. alcohol consumption. The WHO osteopenia and osteoporosis criteria consider spine, hip or forearm BMD measures by DEXA and compare this to the youngadult BMD mean [37]. Here we assessed BMD using CUBA which is a cheaper, safer and relatively precise alternative to DEXA, although can be limited by poor foot positioning $[24,40]$. We compared the sex-specific mean which was in older, rather than younger, adults where peak BMD will have already deteriorated, potentially underestimating the number of participants with low or very low BMD [3, 41]. Furthermore, use of CUBA estimates BMD in the calcaneus, rather than the spine, hip or forearm [37]. Although calibration of devices were regularly checked and measurements taken at least twice in each foot [25], random measurement error is possible. Despite these limitations, using a CUBA devise is an inexpensive and accurate method to evaluate BMD, without any radiation exposure [12]. Time between PCS and BUA assessment varied among participants, with the shortest interval being 18 months. This relatively short time interval in some participants will result in an estimation of what current/near future BMD is, rather than predicting BMD status several years in advance.

\section{Further research}

The use of PCS score as a predictor of BMD in later life warrants further investigation. Despite having a different function to the FRAX tool, direct comparison between PCS score and FRAX is required to evaluate the effectiveness of each.
SF-36 has the potential to predict many health outcomes, therefore may prove to be a useful tool in clinical practice.

\section{Conclusion}

Study findings indicate that self-reported physical functioning is a tool capable of predicting future BMD and identifying at-risk individuals in an apparently healthy population, especially in women. In the current climate, where increased sedentary activity and a reduction of routine medical appointments due to COVID-19 will have implications on bone health, self-reported functional health may prove a useful and inexpensive indicator to stratify populations by risk of low BMD. Further validation is required to gain insight into the role of PCS score in clinical practice.

Acknowledgements We are grateful to all the participants who have been part of the project and to the many members of the study teams at the University of Cambridge who have enabled this research.

Author contribution KM, VK and PKM contributed to the study conception and KM and PKM supervised SP. SP performed literature review and statistical analysis. KTK and NJW are PIs of EPIC-Norfolk. The manuscript was written by SP and all authors read and reviewed the final manuscript.

Funding This work was supported by funding from the Medical Research Council (Grant/Award Numbers: MR/N003284/1, MCUU_12015/1) and Cancer Research UK (Grant/Award Number: C864/ A14136), with additional support from the Stroke Association, British Heart Foundation, Department of Health, Europe Against Cancer Programme Commission of the European Union, Food Standards Agency, Research into Ageing, Academy of Medical Sciences and Wellcome Trust. Sarah Perrott received an institutional grant from the University of Aberdeen Academic Centre for Applied Clinical and Translational Research into Ageing (ACTRA). The role of this grant was to financially support a student researcher from November 2020 to January 2021.

Availability of data and materials There are no linked research data sets for this paper. Data will be made available on request to the steering committee of EPIC-Norfolk.

\section{Declarations}

Ethics approval The EPIC-Norfolk study was approved by the Norwich Research Ethics Committee.

Conflicts of interest None.

\section{References}

1. Henriques-Neto D, Magalhães JP, MH-R, Diana A. Santos, PhD, Fátima Baptista and LBS, (2020) Physical fitness and bone health in young athletes and nonathletes. Sports Health 12:441-448. https://doi.org/10.1177/1941738120931755 
2. Sözen T, Özışık L, Başaran NÇ (2017) An overview and management of osteoporosis. Eur J Rheumatol 4:46-56. https://doi.org/ 10.5152/eurjrheum. 2016.048

3. Troy KL, Mancuso ME, Butler TA, Johnson JE (2018) Exercise early and often: effects of physical activity and exercise on women's bone health. Int J Environ Res Public Health 15:878. https:// doi.org/10.3390/ijerph15050878

4. Dempster DW (2011) Osteoporosis and the burden of osteoporosis-related fractures. Am J Manag Care 17(Suppl 6):164-169

5. Weinstein SL, Ponseti I V (2016) The burden of musculoskeletal conditions. J Bone Jt Surg 98(16):1331. https://doi.org/10.2106/ JBJS.16.00595

6. Jayne K, Sale EC (2017) Exercise and bone health across the lifespan. Biogerontology 18:931-946. https://doi.org/10.1007/ s10522-017-9732-6

7. Tsai J-T, Hou M-F, Chen Y-M, Wan TT, H; Kao, Hao-Yun; Shi H-Y, (2013) Predicting quality of life after breast cancer surgery using ANN-based models: performance comparison with MR. Support Care Cancer 21:1341-1350

8. Rumsfeld JS, MaWhinney S, McCarthy M, Jr; Shroyer, A L; VillaNueva, C B; O'Brien, M; Moritz, T E; Henderson, W G; Grover, F L; Sethi, G K; Hammermeister KE, (1999) Health-related quality of life as a predictor of mortality following coronary artery bypass graft surgery. Participants of the Department of Veterans Affairs Cooperative Study Group on Processes, Structures, and Outcomes of Care in Cardiac Surgery. JAMA 281:1298-1303

9. Schron E, Friedmann E, Thomas S (2014) Does health-related quality of life predict hospitalization or mortality in patients with atrial fibrillation? J Cardiovasc Electrophysiol 25:23-28

10. Chen Y-J, Tu H-P, Lee C-L, Huang W-C, Yang J-S, Li C-F, Chen C-H, Lin K-L (2019) Comprehensive exercise capacity and quality of life assessments predict mortality in patients with pulmonary arterial hypertension. Acta Cardiol Sin 35:55-64

11. Watts NB (2018) Postmenopausal osteoporosis: a clinical review. J Women's Heal 27.https://doi.org/10.1089/jwh.2017.6706

12. Lane NE (2006) Epidemiology, etiology, and diagnosis of osteoporosis. Am J Obstet Gynecol 194.https://doi.org/10.1016/j.ajog.2005.08.047

13. Watson SL, Weeks BK, Weis LJ et al (2018) High-intensity resistance and impact training improves bone mineral density and physical function in postmenopausal women with osteopenia and osteoporosis: the LIFTMOR randomized controlled trial. J Bone Miner Res 33:211-220. https://doi.org/10.1002/jbmr.3284

14. Pande I, Scott D, O'Neill T et al (2006) Quality of life, morbidity and mortality after low trauma hip fracture in men. Ann Rheum Dis 65:87-92

15. Center J, Nguyen T, Schneider D (1999) Mortality after all major types of osteoporotic fracture in men and women: an observational study. Lancet 9156:878-882

16. Taaffe DR, Simonsick EM, Visser M et al (2003) Lower extremity physical performance and hip bone mineral density in elderly black and white men and women: cross-sectional associations in the health ABC study. Journals Gerontol - Ser A Biol Sci Med Sci 58:934-942. https://doi.org/10.1093/gerona/58.10.m934

17. Harvey NC, Od A, Orwoll E et al (2018) Measures of physical performance and muscle strength and aBMD: a meta-analysis of the osteoporotic fractures in men (MrOS) study. Am Soc Bone Miner Res 33:2150-2157. https://doi.org/10.1002/jbmr.3556

18. Hopman WM, Berger C, Joseph L et al (2019) Longitudinal assessment of health-related quality of life in osteoporosis: data from the population-based Canadian multicentre osteoporosis study. Osteoporos Int 30:1635-1644. https://doi.org/10.1007/ s00198-019-05000-y

19. Dennison EM, Syddall HE, Statham C et al (2006) Relationships between SF-36 health profile and bone mineral density: the Hertfordshire cohort study. Osteoporos Int 17:1435-1442. https://doi. org/10.1007/s00198-006-0151-9
20. Day N, Oakes S, Luben R, Khaw KT, Bingham S, Welch AWN (1999) EPIC-Norfolk: study design and characteristics of the cohort. European Prospective Investigation of Cancer. Br J Cancer 80:95-103

21. Fawkes C (2013) SF-36: National Council for Osteopathic Research. https://www.ncor.org.uk/wp-content/uploads/2013/01/ SF-36.pdf. Accessed 3 Jan 2021

22. Brazier E, Harper R, Jones NMB et al (1992) Validating the SF-36 health survey questionnaire: new outcome. Gen Pract 305:160-164

23. (2021) The EPIC-Norfolk study: second health check (2HC). In: Univ. Cambridge. https://www.epic-norfolk.org.uk/about-epicnorfolk/health-checks/second-health-check-2hc/. Accessed 6 Mar 2021

24. He YQ, Fan B, Hans D et al (2000) Assessment of a new quantitative ultrasound calcaneus measurement: precision and discrimination of hip fractures in elderly women compared with dual X-ray absorptiometry. Osteoporos Int 11:354-360. https://doi.org/10. 1007/s001980070125

25. Jakes RW, Khaw K, Day NE et al (2001) Primary care by heel bone among Norfolk cohort of European population based study. Br Med J 322:1-5

26. Welch A, Camus ÆJ, Dalzell ÆN et al (2004) Broadband ultrasound attenuation (BUA) of the heel bone and its correlates in men and women in the EPIC-Norfolk cohort: a cross-sectional population-based study. Osteoporos Int 15:217-225. https://doi. org/10.1007/s00198-003-1410-7

27. Dobbs MB, Buckwalter J, Saltzman C (1999) Osteoporosis: the increasing role of the orthopaedist. Iowa Orthop J 19:43-52

28. Lohman T, Roche A, Martorell R (1991) Anthropometric standardization reference manual. Human Kinetics Books, Champaign, IL

29. Jordan H, Roderick P, Martin D (2004) The index of multiple deprivation 2000 and accessibility effects on health. J Epidemiol Community Heal 58:250-257. https://doi.org/10.1136/jech.2003. 013011

30. Du Y, Zhao LJ, Xu Q et al (2017) Socioeconomic status and bone mineral density in adults by race/ethnicity and gender: the Louisiana osteoporosis study. Osteoporos Int 28:1699-1709. https:// doi.org/10.1007/s00198-017-3951-1

31. Brennan SL, Winzenberg TM, Pasco JA et al (2013) Social disadvantage, bone mineral density and vertebral wedge deformities in the Tasmanian older adult cohort. Osteoporos Int 24:1909-1916. https://doi.org/10.1007/s00198-012-2211-7

32. Alfred T, Ben-Shlomo Y, Cooper R et al (2013) Genetic markers of bone and joint health and physical capability in older adults: the HALCyon programme. Bone 52:2013. https://doi.org/10.1016/j. bone.2012.10.004

33. Ahmeidat A, Bhattacharya S, Luben RN et al (2021) Long-term effects of gestational diabetes on bone mineral density and fracture risk: analysis of the Norfolk cohort of the European Prospective Investigation into Cancer (EPIC-Norfolk) population-based study. Maturitas 144:68-73

34. Julian C, Lentjes MAH, Huybrechts I et al (2016) Fracture risk in relation to serum 25-hydroxyvitamin D and physical activity: results from the EPIC-Norfolk cohort study. PLoS One 1-16.https://doi.org/10.1371/journal.pone.0164160

35. Pedro BJ, Hetherington-rauth M, Sardinha B (2020) Sedentary patterns are associated with bone mineral density and physical function in older adults: cross-sectional and prospective data. Int J Environ Res Public Health 17

36. Finkelstein JS, Brockwell SE, Mehta V et al (2008) Bone mineral density changes during the menopause transition in a multiethnic cohort of women. J Clin Endocrinol Metab 93:861-868. https:// doi.org/10.1210/jc.2007-1876 
37. Karaguzel G, Holick MF (2010) Diagnosis and treatment of osteopenia. Rev Endocr Metab Disord 11:237-251. https://doi.org/10. 1007/s11154-010-9154-0

38. Black DM, Steinbuch M, Palermo L et al (2001) An assessment tool for predicting fracture risk in postmenopausal women. Osteoporos Int 12:519-528

39. Surtees P, Wainwright N, Khaw K (2004) Obesity, confidant support and functional health: cross-sectional evidence from the EPIC-Norfolk cohort. Int J Obes Relat Metab Disord 28:748-758. https://doi.org/10.1038/sj.ijo.0802636
40. Diessel E, Fuerst T, Njeh CF et al (2000) Comparison of an imaging heel quantitative ultrasound device (DTU-one) with densitometric and ultrasonic measurements. Br J Radiol 73:23-30. https://doi.org/10.1259/bjr.73.865.10721316

41. Boskey AL, Coleman R (2010) Critical reviews in oral biology \& medicine: aging and bone. J Dent Res 89:1333-1348. https://doi. org/10.1177/0022034510377791

Publisher's note Springer Nature remains neutral with regard to jurisdictional claims in published maps and institutional affiliations. 